\title{
Anti-trees and right-angled Artin subgroups of braid groups
}

\author{
SANG-HYUN KIM \\ THOMAS KOBERDA
}

\begin{abstract}
We prove that an arbitrary right-angled Artin group $G$ admits a quasi-isometric group embedding into a right-angled Artin group defined by the opposite graph of a tree, and, consequently, into a pure braid group. It follows that $G$ is a quasi-isometrically embedded subgroup of the area-preserving diffeomorphism groups of the 2-disk and of the 2-sphere with $L^{p}$-metrics for suitable $p$. Another corollary is that there exists a closed hyperbolic manifold group of each dimension which admits a quasi-isometric group embedding into a pure braid group. Finally, we show that the isomorphism problem, conjugacy problem, and membership problem are unsolvable in the class of finitely presented subgroups of braid groups.
\end{abstract}

20F36; 53D05, 20F10, 20F67

\section{Introduction}

A right-angled Artin group on a finite graph $\Gamma$ is a group generated by the vertices of $\Gamma$ with commutation relations defined by the adjacency relations in $\Gamma$. In this article, we adopt the opposite of the usual convention used for discussing right-angled Artin groups, and we write

$$
\left.G(\Gamma)=\langle V(\Gamma)|\left[v_{i}, v_{j}\right]=1 \text { if and only if }\left\{v_{i}, v_{j}\right\} \notin E(\Gamma)\right\rangle .
$$

Here, $V(\Gamma)$ and $E(\Gamma)$ denote the set of vertices and edges of $\Gamma$.

\subsection{Main results}

For two groups $G$ and $H$ equipped with metrics, a quasi-isometric group embedding from $G$ to $H$ (with quasi-isometry constant $C$ ) is an injective group homomorphism $f: G \rightarrow H$ such that the following estimate holds for every $x$ and $y$ in $G$ :

$$
d_{G}(x, y) / C-C \leq d_{H}(f(x), f(y)) \leq C d_{G}(x, y)+C .
$$


We will also say that $G$ is a quasi-isometrically embedded subgroup of $H$. We will endow each finitely generated group with a word-metric. Note that every quasi-isometric group embedding between finitely generated groups is bi-Lipschitz.

The principal result of this article is the following:

Theorem 1.1 For each finite graph $\Gamma$, there exists a finite tree $T$ such that $G(\Gamma)$ admits a quasi-isometric group embedding into $G(T)$.

In the usual convention for describing right-angled Artin groups, the tree $T$ should be replaced with its opposite graph $T^{\text {opp }}$, a graph which could reasonably be called an anti-tree. The tree $T$ in Theorem 1.1 is not produced in an ad hoc manner, but is rather built as a subtree of the universal cover of $\Gamma$.

Let us summarize some consequences of the main theorem. The reader is referred to the last section for more details and background.

We write $B_{n}$ for the braid group on $n$ strands, which is identified with the mapping class group of the $n$-punctured disk $D_{n}$ fixing the boundary pointwise. The pure braid group $P_{n}<B_{n}$ is the kernel of the natural puncture permutation representation

$$
B_{n} \rightarrow S_{n}<\operatorname{Aut}\left(H_{1}\left(D_{n}, \mathbb{Z}\right)\right) .
$$

We let $\operatorname{Symp}\left(D^{2}, \partial D^{2}\right)$ be the group of area-preserving diffeomorphisms (symplectomorphisms) of the unit 2-disk that are the identity in a neighborhood of the boundary. We endow $\operatorname{Symp}\left(D^{2}, \partial D^{2}\right)$ with the $L^{p}$-metric $d_{p}$; see Sections 4.2 and 4.3. Crisp and Wiest [18] proved that the right-angled Artin group on each planar graph (more generally, each planar-type graph) admits quasi-isometric group embeddings into $P_{n}$ for some $n$, and into $\left(\operatorname{Symp}\left(D^{2}, \partial D^{2}\right), d_{2}\right)$. They asked whether or not an arbitrary right-angled Artin group embeds into $P_{n}$ for some $n$; see Crisp and Wiest [17].

We denote by $\operatorname{Symp}\left(S^{2}\right)$ the area-preserving diffeomorphism group of the unit 2sphere $S^{2}$, again equipped with the $L^{p}$-metric. Note that $\operatorname{Symp}\left(S^{2}\right)$ is equal to the group of Hamiltonian symplectomorphisms on $S^{2}$ [33, Section 1.4.H]. M Kapovich showed that every right-angled Artin group embeds into $\operatorname{Symp}\left(S^{2}\right)$, asking whether or not this embedding can be chosen to be quasi-isometric with respect to $L^{2}$-metric [24]. We generalize the result of Crisp and Wiest, and give an affirmative answer to the $L^{p}$-version of Kapovich's question for $p>2$.

Corollary 1.2 Every right-angled Artin group admits quasi-isometric group embeddings into the following groups:

(1) $P_{n}$ for some $n$.

(2) $\operatorname{Symp}\left(D^{2}, \partial D^{2}\right)$ with $L^{p}$-metric for $1 \leq p \leq \infty$.

(3) $\operatorname{Symp}\left(S^{2}\right)$ with $L^{p}$-metric for $2<p \leq \infty$. 
Although largely influenced by their papers [18; 24], our proof does not rely on the above-mentioned results of Crisp and Wiest or Kapovich. In Corollary 1.2(1), our analysis tells us that the number of strands $n$ can be chosen so that $\log _{2} \log _{2} n \leq m^{2}$, where $m$ is the number of the generators of the right-angled Artin group.

Corollary 1.2(1) can be applied to recover an algebraic result of Baudisch concerning the structure of two-generated subgroups of right-angled Artin groups:

Corollary 1.3 (See Baudisch [3]) Every two-generated subgroup of a right-angled Artin group is either abelian or free.

A group $G$ is called special if $G=\pi_{1}(X)$ for some compact CAT(0) cube complex $X$ that locally isometrically embeds into the Salvetti complex of a right-angled Artin group. If a group has a finite-index special subgroup, it is called virtually special (see Haglund and Wise [22]). The class of virtually special groups is surprisingly large, including all the finite-volume hyperbolic 3-manifold groups; see [22], Wise [35], and Agol [1].

Corollary 1.4 A special group admits a quasi-isometric group embedding into a pure braid group.

In particular, every finite-volume hyperbolic 3-manifold group is virtually a quasiisometrically embedded subgroup of a pure braid group. Theorem 1.1 also furnishes examples of quasi-isometrically embedded higher-dimensional closed hyperbolic manifold subgroups of a pure braid group, which to the authors' knowledge are the first such examples.

Corollary 1.5 For each $n \geq 2$, there exists an $n$-dimensional closed hyperbolic manifold whose fundamental group admits a quasi-isometric group embedding into a pure braid group.

In particular, we will see that the fundamental group of a manifold cover of the 4dimensional all-right hyperbolic 120-cell orbifold quasi-isometrically embeds into a pure braid group. This answers a question posed by Crisp and Wiest in [18].

Recall that the isomorphism problem for a class $\mathfrak{C}$ of finitely presented groups asks to find an algorithm which, on an input of two members $A, B \in \mathfrak{C}$, halts and determines whether or not $A \cong B$. In [9], Bridson uses right-angled Artin subgroups of mapping class groups to show that the isomorphism problem is unsolvable when $\mathfrak{C}$ is the class of finitely presented subgroups of a sufficiently high-genus surface mapping class group. Theorem 1.1 allows us to replace the surface mapping class group with a braid group: 
Corollary 1.6 Let $\mathfrak{C}_{n}$ be the class of finitely presented subgroups of the planar braid group $B_{n}$. If $n$ is sufficiently large then the isomorphism problem for $\mathfrak{C}_{n}$ is unsolvable.

Recall that the conjugacy problem in a finitely presented group $H$ asks whether there exists an algorithm which, on an input of two elements $g, h \in H$, halts and determines whether or not $g$ and $h$ are conjugate to each other in $H$. The membership problem for a subgroup $K<H$ asks whether there exists an algorithm which, on an input of a word $w$ in the generators of $H$, halts and determines whether or not the element of $H$ represented by $w$ lies in $K$. With this setup, Theorem 1.1 has the following consequence for braid groups:

Corollary 1.7 For all $n$ sufficiently large, there exists a finitely presented subgroup $H<B_{n}$ such that the conjugacy problem for $H$ is unsolvable and for which the membership problem is unsolvable.

The word problem is solvable among all finitely presented subgroups of braid groups by virtue of their residual finiteness.

\subsection{Notes and references}

In [24], M Kapovich shows that every right-angled Artin group $G(\Gamma)$ embeds in the group of hamiltonian symplectomorphisms of the 2-sphere. In the second part of his proof, Kapovich resolves technical difficulties arising from right-angled Artin groups on non-planar graphs. As trees are planar, Theorem 1.1 simplifies Kapovich's argument. We will also strengthen Kapovich's theorem to Corollary 1.2(3).

Right-angled Artin subgroups of right-angled Artin groups and of mapping class groups have been studied by various authors from several points of view. The reader may consult Kim [26], Clay, Leininger and Mangahas [14], Crisp and Paris [16], Crisp and Wiest [18], Kim and Koberda [27; 28; 29] and Koberda [31], for instance.

Mapping class groups have proven to be a fruitful setting for the study of right-angled Artin groups for their own sake. The setup which most easily lends itself to analysis is when one has a homomorphism

$$
\phi: G(\Gamma) \rightarrow \operatorname{Mod}(S)
$$

such that $\phi$ maps each vertex generator of $G(\Gamma)$ to a mapping class with a connected support $[14 ; 31]$. When the support of a mapping class is disconnected, various algebraic pathologies may arise (see [31], for instance).

In the right-angled Artin group setting, the analogue of a mapping class with disconnected support is an element $g$ of a right-angled Artin group that is not a pure factor, 
ie an element $1 \neq g \in G(\Gamma)$ which can be written as a product $g=g_{1} \cdot g_{2}$ of non-trivial elements with no common powers such that $\left[g_{1}, g_{2}\right]=1$. If

$$
\phi: G(\Lambda) \rightarrow G(\Gamma)
$$

is a homomorphism of right-angled Artin groups where vertex generators of $G(\Lambda)$ are sent to elements of $G(\Gamma)$ which are not pure factors, the same algebraic pathologies which occur in the mapping class group setting can again occur.

Oftentimes, various fortuitous circumstances allow one to circumvent the algebraic pathologies which occur when studying homomorphisms from a right-angled Artin group to another right-angled Artin group, or to a mapping class group. Such analyses have been carried out in Casals-Ruiz, Duncan and Kazachkov [12] and Kim and Koberda [30] in the right-angled Artin group and mapping class group cases, respectively, for instance.

This article is entirely concerned with injective maps $G(\Gamma) \rightarrow G(T)$ where vertex generators of $G(\Gamma)$ are sent to elements of $G(T)$ which are never pure factors, and with compositions of such maps with injective homomorphisms $G(T) \rightarrow P_{n}$, so that the vertex generators of $G(\Gamma)$ are always sent to mapping classes with disconnected support.

Let us comment on the precursors of Theorem 1.1. Crisp and Wiest proved that $G(\Gamma)$ embeds into $G(\Lambda)$ if $\Lambda$ is a finite cover of $\Gamma$ [17, Proposition 19], and M Kapovich extended this result to the case when $\Lambda$ is a finite orbi-cover of $\Gamma$ [24, Lemma 2.3]. The methods of this paper were also inspired by the analysis in [12] of an injective map $G\left(C_{5}\right) \rightarrow G\left(L_{8}\right)$, where $C_{5}$ denotes the cycle on five vertices and where $L_{8}$ denotes a path on eight vertices.

Acknowledgements Kim is supported by the Basic Science Research Program through the National Research Foundation of Korea (NRF) funded by the Ministry of Education, Science and Technology (2013R1A1A1058646). Kim is also supported by Samsung Science and Technology Foundation (SSTF-BA1301-06). Koberda is partially supported by NSF grant DMS-1203964. The authors are grateful to the referee for several helpful comments.

\section{Preliminaries}

Every graph in this paper will be assumed to be simplicial. For two graphs $X$ and $Y$, we write $X \leq Y$ if a graph $X$ is an induced subgraph of another graph $Y$. This means that $X$ is a subgraph of $Y$ such that

$$
E(X)=\left(\begin{array}{c}
V(X) \\
2
\end{array}\right) \cap E(Y) .
$$


If $S$ is a subset of $V(Y)$, we often identify $S$ with the induced subgraph of $Y$ on $S$. For a vertex $x$ in a graph $X$, the link and the star of $v$ are defined respectively as:

$$
\operatorname{Lk}(v)=\{u \in V(X) \mid\{u, v\} \in E(X)\}, \quad \operatorname{St}(v)=\operatorname{Lk}(v) \cup\{v\} .
$$

Let $\Gamma$ be a finite graph. Each element in $V(\Gamma) \cup V(\Gamma)^{-1}$ is called a letter in $G(\Gamma)$. A word in $G(\Gamma)$ is a finite sequence of letters, and usually written as a multiplication of letters. The word length of an element $g \in G(\Gamma)$ is the length of a shortest word representing $g$ and denoted as $\|g\|$. A word $w$ is reduced if its length realizes the word length. The support of $g \in G(\Gamma)$ is the set of the vertices $v$ such that $v$ or $v^{-1}$ appears in a reduced word for $g$. The support of $g$ is denoted as $\operatorname{supp}(g)$. For example, $\operatorname{supp}\left(a^{-1} b b^{-1}\right)=\{a\}$ if $a$ and $b$ are vertices.

Let $w$ be a (possibly non-reduced) word in $G(\Gamma)$ and $v$ be a vertex of $\Gamma$. A cancellation of $v$ in $w$ is a subword

$$
v^{ \pm 1} w^{\prime} v^{\mp 1}
$$

of $w$ such that $\operatorname{supp}\left(w^{\prime}\right) \cap \operatorname{Lk}(v)=\varnothing$. If, furthermore, no letters in $w^{\prime}$ are equal to $v$ or $v^{-1}$ then we say the word

$$
v^{ \pm 1} w^{\prime} v^{\mp 1}
$$

is an innermost cancellation of $v$ in $w$. If $v$ or $v^{-1}$ is a letter in $w$ and $v \notin \operatorname{supp} w$, then there is a cancellation of $v$ in $w$, and hence there is an innermost one; this follows from a well-known solution to the word problem in right-angled Artin groups [36; 13]. Hence, every non-reduced word contains an innermost cancellation. If there are no cancellations of a vertex $v$ in $w$, then $v$ "survives" in each reduced word $w^{\prime}$ representing $w$, in the sense that the number of occurrences of $v$ or $v^{-1}$ in $w$ is the same as that in $w^{\prime}$.

\section{Proof of Theorem 1.1}

For the purposes of Theorem 1.1, we may assume that $\Gamma$ is connected. For otherwise we can write $\Gamma=\Gamma_{1} \sqcup \Gamma_{2}$, so that $G(\Gamma)=G\left(\Gamma_{1}\right) \times G\left(\Gamma_{2}\right)$. If there are trees $T_{1}, T_{2}$ and a quasi-isometric group embedding from $G\left(\Gamma_{i}\right)$ into $G\left(T_{i}\right)$ for each $i=1$, 2 , then we simply let $T$ be the tree obtained by joining a vertex in $T_{1}$ to another vertex in $T_{2}$ by a length-two path. Since there is a natural isometric embedding from $G\left(T_{1}\right) \times G\left(T_{2}\right)$ into $G(T)$, we have a quasi-isometric group embedding from $G(\Gamma)$ into $G(T)$.

From now on, we will let $\Gamma$ be a finite, connected graph and $p: \widetilde{\Gamma} \rightarrow \Gamma$ be its universal cover. We will fix an arbitrary order on $V(\widetilde{\Gamma})$. Every induced subgraph of $\widetilde{\Gamma}$ is a forest. 
Let $T$ be a finite induced subgraph of $\widetilde{\Gamma}$. Then $T$ induces a group homomorphism $\phi(\Gamma, T): G(\Gamma) \rightarrow G(T)$, defined by

$$
\phi(\Gamma, T): v \mapsto \prod_{t \in p^{-1}(v) \cap T} t,
$$

where we define this product to be the identity if the indexing set is empty. Since no two vertices in $p^{-1}(v)$ are adjacent, this product is well-defined.

Suppose $w$ is a word in $G(\Gamma)$ written as $x_{1}^{e_{1}} x_{2}^{e_{2}} \cdots x_{\ell}^{e_{\ell}}$ for $x_{1}, x_{2}, \ldots, x_{\ell} \in V(\Gamma)$ and $e_{1}, e_{2}, \ldots, e_{\ell}= \pm 1$. Let us write the product

$$
\phi(\Gamma, T)\left(x_{i}\right)=\prod_{t \in p^{-1}\left(x_{i}\right) \cap T} t
$$

in increasing order. We define the $\phi(\Gamma, T)$-homomorphic word of $w$ as the word

$$
\prod_{i=1}^{\ell}\left(\phi(\Gamma, T)\left(x_{i}\right)\right)^{e_{i}} .
$$

Note that this (possibly non-reduced) word represents $\phi(\Gamma, T)(w)$ in $G(T)$.

Definition 3.1 Let $T$ be a finite induced subgraph of $\widetilde{\Gamma}$ and $F \subseteq V(T)$. We say $\phi(\Gamma, T)$ is $F$-surviving if, for every reduced word $w$ in $G(\Gamma)$ and $v \in F$, the $\phi(\Gamma, T)-$ homomorphic word of $w$ does not have a cancellation of $v$.

We make some immediate observations:

Lemma 3.2 Let $T^{\prime}$ be a finite induced forest in $\widetilde{\Gamma}$ and $T \leq T^{\prime}$.

(1) For each $w \in G(\Gamma)$, we have $\operatorname{supp}(\phi(\Gamma, T)(w)) \subseteq \operatorname{supp}\left(\phi\left(\Gamma, T^{\prime}\right)(w)\right)$. In particular, $\operatorname{ker} \phi\left(\Gamma, T^{\prime}\right) \leq \operatorname{ker} \phi(\Gamma, T)$.

(2) Suppose $F^{\prime} \subseteq F \subseteq V(T)$. If $\phi(\Gamma, T)$ is $F$-surviving, then $\phi\left(\Gamma, T^{\prime}\right)$ is $F^{\prime}$ surviving.

(3) For $F_{1}, F_{2} \subseteq V(T)$, if $\phi(\Gamma, T)$ is $F_{1}$-surviving and $F_{2}$-surviving, then $\phi(\Gamma, T)$ is $\left(F_{1} \cup F_{2}\right)$-surviving.

Proof There is a natural quotient map $\eta: G\left(T^{\prime}\right) \rightarrow G(T)$ sending the vertices in $T^{\prime} \backslash T$ to the identity. Since $\operatorname{supp} \eta(w) \subseteq \operatorname{supp}(w)$ for $w \in G\left(T^{\prime}\right)$ and $\phi(\Gamma, T)=\eta \circ \phi\left(\Gamma, T^{\prime}\right)$, we obtain (1). The parts (2) and (3) are clear from definitions.

Lemma 3.3 Let $T$ be a finite subtree of $\widetilde{\Gamma}$ and $F \subseteq V(T)$ such that $p(F)=V(\Gamma)$. If $\phi(\Gamma, T)$ is $F$-surviving then $\phi(\Gamma, T)$ is a quasi-isometric group embedding. 
Proof Let $w$ be a reduced word in $G(\Gamma)$. Note that $\|\phi(\Gamma, T)(w)\| \leq|V(T)|\|w\|$. On the other hand, the $F$-surviving condition implies that some reduced word for $\phi(\Gamma, T)(w)$ contains the $\phi(\Gamma, F)$-homomorphic word of $w$ as a subsequence. This gives $\|\phi(\Gamma, T)(w)\| \geq\|w\|$, and, in particular, $\phi(\Gamma, T)$ is injective.

If $v$ is a vertex of $\Gamma$, we denote by $\Gamma \backslash v$ the induced subgraph of $\Gamma$ on $V(\Gamma) \backslash\{v\}$. The following lemma is a key inductive step in the proof of Theorem 3.5.

Lemma 3.4 Let $v$ be a vertex of $\Gamma$ and $T_{0}$ be a finite tree in $\widetilde{\Gamma}$. If the restriction of $\phi\left(\Gamma, T_{0}\right)$ to $G(\Gamma \backslash v)$ is injective, then for each vertex $v^{\prime} \in p^{-1}(v)$ there exists a finite tree $T$ in $\widetilde{\Gamma}$ containing $T_{0} \cup\left\{v^{\prime}\right\}$ such that $\phi(\Gamma, T)$ is $v^{\prime}$-surviving and injective.

Proof Choose a finite tree $T_{1} \leq \widetilde{\Gamma}$ containing $T_{0} \cup \operatorname{St}\left(v^{\prime}\right)$. Let $\Sigma$ be the set of deck transformations $\sigma: \widetilde{\Gamma} \rightarrow \widetilde{\Gamma}$ such that $\sigma\left(T_{1}\right) \cap T_{1} \neq \varnothing$. This set $\Sigma$ is finite since the deck transformation group of $\widetilde{\Gamma}$ over $\Gamma$ acts freely and simplicially. Define

$$
T=\bigcup_{\sigma \in \Sigma} \sigma\left(T_{1}\right)
$$

We first claim that $\phi(\Gamma, T)$ is $v^{\prime}$-surviving. For this, let us suppose $w$ is a nontrivial reduced word in $G(\Gamma)$ such that the $\phi(\Gamma, T)$-homomorphic word of $w$ has an innermost cancellation of $v^{\prime}$. We then find a subword

$$
v^{ \pm 1} w_{1} v^{\mp 1}
$$

of $w$ such that $v \notin \operatorname{supp} w_{1}$ and

$$
\operatorname{supp}\left(\phi(\Gamma, T)\left(w_{1}\right)\right) \cap \operatorname{Lk}_{\widetilde{\Gamma}}\left(v^{\prime}\right)=\varnothing .
$$

Since $v^{ \pm 1} w_{1} v^{\mp 1}$ is reduced, there exists a vertex $x \in \operatorname{supp} w_{1} \cap \operatorname{Lk}_{\Gamma}(v)$. Then

$$
p^{-1}(x) \cap \operatorname{Lk}_{\widetilde{\Gamma}}\left(v^{\prime}\right)=\left\{x^{\prime}\right\} \subseteq \operatorname{Lk}_{\widetilde{\Gamma}}\left(v^{\prime}\right) \subseteq T_{1}
$$

for some vertex $x^{\prime}$. Since $x^{\prime} \notin \operatorname{supp} \phi(\Gamma, T)\left(w_{1}\right)$, there exists an innermost cancellation of $x^{\prime}$ in the $\phi(\Gamma, T)$-homomorphic word of $w_{1}$. This implies that $w_{1}$ has a subword

$$
x^{ \pm 1} w_{2} x^{\mp 1}
$$

such that

$$
\operatorname{supp}\left(\phi(\Gamma, T)\left(w_{2}\right)\right) \cap \operatorname{Lk}_{\widetilde{\Gamma}}\left(x^{\prime}\right)=\varnothing .
$$

The restriction of $\phi\left(\Gamma, T_{1}\right)$ to $G(\Gamma \backslash v)$ is injective by the assumption and Lemma 3.2(1). In particular,

$$
\phi\left(\Gamma, T_{1}\right)\left(x^{ \pm 1} w_{2} x^{\mp 1}\right) \neq \phi\left(\Gamma, T_{1}\right)\left(w_{2}\right) .
$$


So we can find vertices

$$
x^{\prime \prime} \in p^{-1}(x) \cap T_{1}, \quad y \in \operatorname{supp}\left(\phi\left(\Gamma, T_{1}\right)\left(w_{2}\right)\right) \cap \operatorname{Lk}_{\widetilde{\Gamma}}\left(x^{\prime \prime}\right) \subseteq T_{1} .
$$

Since $x^{\prime}, x^{\prime \prime} \in p^{-1}(x) \cap T_{1}$, there is a deck transformation $\sigma \in \Sigma$ such that $\sigma\left(x^{\prime \prime}\right)=x^{\prime}$. Note that

$$
\sigma(y) \in \operatorname{supp}\left(\phi\left(\Gamma, \sigma\left(T_{1}\right)\right)\left(w_{2}\right)\right) \cap \operatorname{Lk}_{\widetilde{\Gamma}}\left(x^{\prime}\right) \subseteq \operatorname{supp}\left(\phi(\Gamma, T)\left(w_{2}\right)\right) \cap \operatorname{Lk}_{\widetilde{\Gamma}}\left(x^{\prime}\right) .
$$

This contradicts $(*)$.

It remains to show that $\phi(\Gamma, T)$ is injective. Suppose $w$ is a reduced word in $\operatorname{ker} \phi(\Gamma, T) \backslash 1$. By the assumption, $v \in \operatorname{supp} w$, and hence the $\phi(\Gamma, T)$-homomorphic word of $w$ contains an innermost cancellation of $v^{\prime}$. This is a contradiction, for $\phi(\Gamma, T)$ is $v^{\prime}$-surviving.

Theorem 1.1 is an immediate consequence of the following theorem.

Theorem 3.5 Let $\Gamma$ be a finite connected graph and $p: \widetilde{\Gamma} \rightarrow \Gamma$ be a universal cover. Then there exists a finite tree $T$ in $\widetilde{\Gamma}$ such that $\phi(\Gamma, T)$ is a quasi-isometric group embedding.

Proof We fix an arbitrary order on $V(\widetilde{\Gamma})$ so that a $\phi(\Gamma, \Lambda)$-homomorphic word is well-defined for each finite subgraph $\Lambda$ of $\widetilde{\Gamma}$. Let $F_{0}$ be a maximal tree in $\Gamma$ and $F$ be a lift of $F_{0}$ in $\widetilde{\Gamma}$, so that $p(V(F))=V(\Gamma)$. Choose a valence-one vertex $v$ in $F_{0}$ and let $v^{\prime}$ be the lift of $v$ in $F$. Note that $\Gamma \backslash v$ is connected and each component of $p^{-1}(\Gamma \backslash v)$ is a universal cover of $\Gamma \backslash v$. Inducting on the number of vertices in $\Gamma$, we may assume that for some finite tree $T_{0}$ contained in $p^{-1}(\Gamma \backslash v) \leq \tilde{\Gamma}$ the map $\phi\left(\Gamma \backslash v, T_{0}\right)$ is a quasi-isometric group embedding. By Lemma 3.4, there is a finite tree $T_{1}$ containing $v^{\prime}$ in $\widetilde{\Gamma}$ such that $\phi\left(\Gamma, T_{1}\right)$ is $v^{\prime}$-surviving and injective. Then for each $u \in V(\Gamma)$, the restriction of $\phi\left(\Gamma, T_{1}\right)$ to $G(\Gamma \backslash u)$ is injective. By a repeated application of Lemmas 3.2 and 3.4, we can enlarge $T_{1}$ to another tree $T \leq \widetilde{\Gamma}$ such that $\phi(\Gamma, T)$ is $F$-surviving. Lemma 3.3 completes the proof.

Remark Let $m=|V(\Gamma)|$. In Lemma 3.4, assuming additionally that $d\left(v^{\prime}, T_{0}\right) \leq 1$, we see that

$$
\left|V\left(T_{1}\right)\right| \leq\left|V\left(T_{0}\right)\right|+\left|\operatorname{St}\left(v^{\prime}\right)\right| \leq\left|V\left(T_{0}\right)\right|+m .
$$

So

$$
|V(T)| \leq|\Sigma|\left|V\left(T_{1}\right)\right| \leq\left|V\left(T_{1}\right)\right|^{3} \leq\left(\left|V\left(T_{0}\right)\right|+m\right)^{3} .
$$

Now let us consider the proof of Theorem 3.5. We can inductively choose $T_{0}$ which contains $F_{0}$, and deduce that

$$
|V(T)| \leq\left(\left(\cdots\left(\left|V\left(T_{0}\right)\right|+m\right)^{3}+m\right)^{3} \cdots+m\right)^{3},
$$


where the iteration is $m$ times. An easy recursive argument shows that

$$
|V(T)| \leq 2^{2^{(m-1)^{2}}} .
$$

\section{Applications}

\subsection{Pure braid groups}

Crisp and Wiest proved that if $\Gamma$ is a planar graph then $G(\Gamma)$ admits a quasi-isometric group embedding into a pure braid group [18]. We give an alternative account of this fact, modulo a general result about faithful homomorphisms from right-angled Artin groups to mapping class groups and their geometric behavior; see [14].

Lemma 4.1 [18] If $\Gamma$ is a planar graph, then for some $n$ we have that $G(\Gamma)$ admits a quasi-isometric group embedding into $P_{n}$.

Let us denote by $\operatorname{PMod}(S)$ the pure mapping class group on the surface $S$. We let $S_{g, n}^{b}$ denote the surface of genus $g$ with $n$ punctures and $b$ boundary components. We write $S_{0, n}$ for $S_{0, n}^{0}$.

Proof Since we have isomorphisms [15, Theorem 8]

$$
P_{n-1} \cong \operatorname{PMod}\left(S_{0, n-1}^{1}\right) \cong \operatorname{PMod}\left(S_{0, n}\right) \times \mathbb{Z},
$$

it suffices to embed $G(\Gamma)$ into $\operatorname{PMod}\left(S_{0, n}\right)$. Embed $\Gamma$ in the sphere and replace each vertex $v$ of $\Gamma$ with a small disk. Deform these disks along the edges of $\Gamma$ to get a configuration of disks $\left\{D_{v}\right\}$ in the sphere which are in bijective correspondence with the vertices of $\Gamma$ and which intersect precisely when the corresponding vertices in $\Gamma$ are adjacent. We require that the boundaries of an overlapping pair of disks intersect at two points, and also that there are no triple intersection of disks.

We now introduce $n$ punctures in the disks as follows: puncture $D_{v}$ in the interior of each intersection with another disk $D_{u}$. Then introduce three punctures in the interior of

$$
D_{v} \backslash \bigcup_{u \neq v} D_{u}
$$

for each $v$, and also three punctures in the exterior of $\bigcup_{v} D_{v}$. It is now easy to produce a pseudo-Anosov mapping class $\psi_{v}$ whose support is the entirety of $D_{v}$. Evidently, the collection $\left\{\psi_{v}\right\} \subset \operatorname{PMod}\left(S_{0, n}\right)$ is a collection of non-trivial mapping classes of the multiply punctured sphere which commute if and only if the corresponding vertices 
are not adjacent in $\Gamma$, and furthermore no two of these mapping classes generate a cyclic subgroup of $\operatorname{PMod}\left(S_{0, n}\right)$. By [14, Theorem 1.1], we see that sufficiently high powers of these mapping classes generate a quasi-isometrically embedded subgroup of $\operatorname{PMod}\left(S_{0, n}\right)$ isomorphic to $G(\Gamma)$.

The proof of Corollary 1.2(1) is immediate from Theorem 1.1 and the preceding lemma. We further claim that if $m=|V(\Gamma)|$, then $G(\Gamma)$ embeds into $P_{n}$ for

$$
n=2^{2^{m^{2}}} \text {. }
$$

To see this, we first embed $G(\Gamma)$ into $G(T)$ for some tree satisfying $|V(T)| \leq 2^{2^{(m-1)^{2}}}$, as in the previous section. Note from the proof of Lemma 4.1 that for $G(T)$ to embed into $P_{n}$ we have only to place three punctures at each vertex and on the exterior, and one puncture at each edge. So it suffices to consider

$$
n \leq|E(T)|+3|V(T)|+3=4|V(T)|+2 .
$$

It follows that, for $m \geq 2$,

$$
n \leq 4 \cdot 2^{2^{(m-1)^{2}}}+2 \leq 2^{2^{m^{2}}}
$$

\subsection{Area-preserving diffeomorphisms of the 2-sphere}

We let $M=S^{2}$ and $\mathcal{G}=\operatorname{Symp}\left(S^{2}\right)$, where $M$ is equipped with the round metric. For $1 \leq p \leq \infty$ and a vector field $X$ on $M$, we define

$$
\|X\|_{p}= \begin{cases}\left(\int_{M}|X|^{p} d x\right)^{1 / p} & \text { if } p<\infty \\ \sup _{x \in M}|X(x)| & \text { if } p=\infty\end{cases}
$$

For each path $\alpha: I \rightarrow \mathcal{G}$, we define the $L^{p}$-length as

$$
\ell_{p}(\alpha)=\int_{0}^{1}\|\partial \alpha / \partial t\|_{p} d t .
$$

Then we have a non-degenerate right-invariant metric on $\mathcal{G}$ given by

$$
d_{p}(\phi, \psi)=\inf \ell_{p}(\alpha),
$$

where the infimum is taken over all paths $\alpha \subseteq \mathcal{G}$ from $\alpha(0)=\phi$ to $\alpha(1)=\psi$. We let $\|\phi\|_{p}=d_{p}(\mathrm{Id}, \phi)$ so that $\|\phi \circ \psi\|_{p} \leq\|\phi\|_{p}+\|\psi\|_{p}$; see [2, Section IV.7.A] and [25, Section II.3.6].

Let us fix a set of distinct points $P=\left\{m_{1}, m_{2}, \ldots, m_{n}\right\}$ in $M$ for some $n$. Choose small disjoint closed disks $D_{1}, D_{2}, \ldots, D_{n}$ such that each $D_{i}$ is centered at $m_{i}$. We 
let $\mathcal{P}_{n}(M)$ be the subgroup of $\mathcal{G}$ consisting of diffeomorphisms which restrict to the identity on $D_{1} \cup D_{2} \cup \cdots \cup D_{n}$. Following [8], we let $X_{n}$ be the configuration space of $n$ distinct points in $M$ with the base point $m=\left(m_{1}, \ldots, m_{n}\right)$. The $n$-strand pure braid group on $M$ is $P_{n}(M)=\pi_{1}\left(X_{n}, m\right)$. If $f \in \mathcal{G}$ and $x=\left(x_{1}, x_{2}, \ldots, x_{n}\right) \in X_{n}$, we let $f(x)$ denote $\left(f\left(x_{1}\right), f\left(x_{2}\right), \ldots, f\left(x_{n}\right)\right)$.

From [34], we have homotopy equivalences

$$
\mathcal{G} \simeq \operatorname{Diff}^{+}(M) \simeq \mathrm{SO}(3)
$$

So the universal cover $p: \widetilde{\mathcal{G}} \rightarrow \mathcal{G}$ is a two-to-one map. Recall that $\widetilde{\mathcal{G}}$ consists of homotopy classes of paths in $\mathcal{G}$. Let $\sigma: I \rightarrow \mathrm{SO}(3)$ denote the rotational isotopy from Id to itself by a full rotation. For each path $\alpha: I \rightarrow \mathcal{G}$ from Id to a map $\phi \in \mathcal{P}_{n}(M)$, we define $\mu_{n}(\alpha) \in P_{n}(M)$ to be the braid represented by $\alpha(I)(m)$. The map $\mu_{n}$ lifts to a map from $p^{-1}\left(\mathcal{P}_{n}(M)\right)$ to $P_{n}(M)$, which is still denoted by $\mu_{n}$. We have a natural commutative diagram

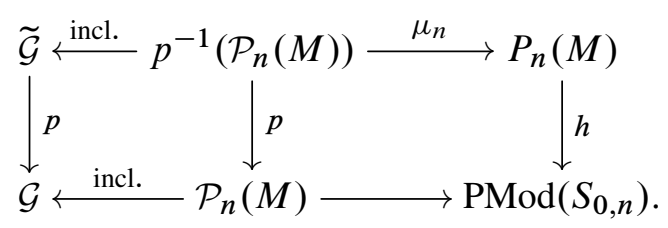

Note that $h$ is the point-pushing map, so $h$ is surjective and ker $h=\mathbb{Z}_{2}$ by the belt trick; see [21, page 251]. The group $\operatorname{PMod}(M \backslash P)=\operatorname{PMod}\left(S_{0, n}\right)$ consists of mapping classes on $M$ fixing each $m_{i}$ pointwise. The groups $P_{n}(M)$ and $\operatorname{PMod}\left(S_{0, n}\right)$ are given with word metrics. Suppose $\phi \in \mathcal{P}_{n}(M)$. We have $p^{-1}(\phi)=\{[\alpha],[\sigma \cdot \alpha]\}$ for some path $\alpha: I \rightarrow \mathcal{G}$ connecting Id to $\phi$. In the above diagram, the square on the right gives

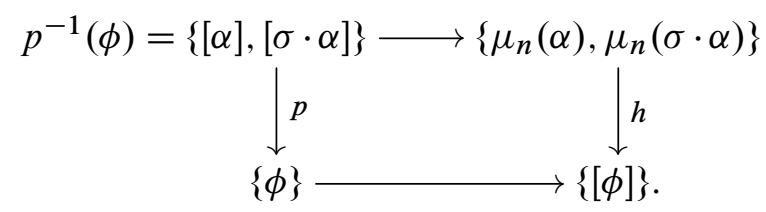

Since $h$ is a quasi-isometry, there exists $C_{0}>0$ such that

$$
\frac{1}{C_{0}}\|[\phi]\|-C_{0} \leq \min \left(\left\|\mu_{n}(\alpha)\right\|,\left\|\mu_{n}(\sigma \cdot \alpha)\right\|\right) .
$$

Brandenbursky and Shelukhin proved that every finitely generated free abelian group quasi-isometrically embeds into $\operatorname{Symp}\left(S^{2}\right)$; see [8]. Using an estimate given in [8], we prove the following lemma.

Lemma 4.2 For $p>2$, there exists $C=C(n, p)>0$ such that, for each $\phi \in \mathcal{P}_{n}(M)$, the word-length $\|[\phi]\|$ in $\operatorname{PMod}(M \backslash P)$ is at most $C\|\phi\|_{p}+C$. 
Proof Let $\phi \in \mathcal{P}_{n}(M)$ and $\alpha: I \rightarrow \mathcal{G}$ be an isotopy from Id to $\phi$. Put

$$
D_{0}=D_{1} \times \cdots \times D_{n} \subseteq X_{n} .
$$

Let $x$ be in the interior of $D_{0}$. We denote by $\gamma(x)$ the component-wise geodesic from $m$ to $x$, and define a loop in $X_{n}$ as

$$
\ell(\alpha, x)=\gamma(x) \cdot \alpha(I)(x) \cdot \gamma(x)^{-1} .
$$

Let $\gamma_{s}(x)$ be the restriction of the path $\gamma(x)$ to $[0, s]$. From a braid isotopy

$$
H(s)=\gamma_{s}(x) \cdot \alpha(I)(\gamma(x)(s)) \cdot\left(\gamma_{s}(x)\right)^{-1},
$$

we see that $[\ell(\alpha, x)]=\mu_{n}(\alpha)$. For the point-pushing map $h$, we have $h\left(\mu_{n}(\alpha)\right)=[\phi]$. Hence,

$$
\frac{1}{C_{0}}\|[\phi]\|-C_{0} \leq\left\|\mu_{n}(\alpha)\right\|=\|[\ell(\alpha, x)]\| .
$$

We see from [8, Lemmas 1 and 2] that for $p>2$ there exists $C_{2}>0$ independent of $\phi$ and $\alpha$ satisfying

$$
\int_{D_{0}}\|[\ell(\alpha, x)]\| d x \leq C_{2} \ell_{p}(\alpha)+C_{2}
$$

Finally, we obtain

$$
\operatorname{vol}\left(D_{0}\right)\left(\frac{1}{C_{0}}\|[\phi]\|-C_{0}\right) \leq \int_{D_{0}}\|[\ell(\alpha, x)]\| d x \leq C_{2} \ell_{p}(\alpha)+C_{2} .
$$

Taking the infimum of the right-hand side, we have a desired inequality.

Let us complete the proof of Corollary 1.2(3). By Theorem 1.1 and Lemma 4.1, every right-angled Artin group $G(\Gamma)$ admits a quasi-isometric group embedding $f_{0}: G(\Gamma) \rightarrow$ $\operatorname{PMod}\left(S_{0, n}\right)$ for some $n$. We can choose $\psi_{v}$ in the proof of Lemma 4.1 to be areapreserving; see [18, Theorem 12] or [2, Lemma III.3.5]. So $f_{0}$ factors through $f_{1}: G(\Gamma) \rightarrow \mathcal{P}_{n}(M)$ for some $f_{1}$. Let $w \in G(\Gamma)$. By Lemma 4.2, there exist $C, C^{\prime}>0$ such that for every $w \in G(\Gamma)$ we have

$$
\frac{1}{C^{\prime}}\|w\|-C^{\prime} \leq\left\|f_{0}(w)\right\|=\left\|\left[f_{1}(w)\right]\right\| \leq C\left\|f_{1}(w)\right\|_{p}+C .
$$

By the following lemma, we see that $f_{1}$ is the desired quasi-isometric group embedding.

Lemma 4.3 Suppose $G$ is a finitely generated torsion-free group with a word-metric $\|\cdot\|$, and $H$ is a group equipped with a non-degenerate right-invariant metric $d$. For 
$h \in H$, we set $\|h\|=d(1, h)$. If there exists $C>0$ and a group homomorphism $\phi: G \rightarrow H$ such that every $g \in G$ satisfies

$$
\|g\| \leq C\|\phi(g)\|+C,
$$

then $\phi$ is a quasi-isometric group embedding.

Proof Suppose $S$ is a finite generating set of $G$ which is used to define the given word-metric. Then for $g=s_{1} \cdots s_{\ell}$, where $s_{i} \in S$ and $\ell=\|g\|$, we have

$$
\|\phi(g)\|=\left\|\prod_{i} \phi\left(s_{i}\right)\right\| \leq \sup _{s \in S}\|\phi(s)\| \cdot\|g\| .
$$

So it suffices to show that $\phi$ is injective. Suppose $\phi(g)=1$. Then for every $n \in \mathbb{Z}$ we have

$$
\left\|g^{n}\right\| \leq C\left\|\phi(g)^{n}\right\|+C=C .
$$

This implies that $\left\{g^{n} \mid n \in \mathbb{Z}\right\}$ is a finite set, and hence $g=1$.

\subsection{Area-preserving diffeomorphisms of the 2-disk}

In order to prove Corollary 1.2(2), we can use the argument in Section 4.2 almost verbatim by substituting $M=D^{2}$ and $\mathcal{G}=\operatorname{Symp}\left(D^{2}, \partial D^{2}\right)$. Here, the $L^{p}$-metric is defined in the same manner. This case is even simpler, as $\widetilde{\mathcal{G}}=\mathcal{G}$ and $P_{n} \cong \operatorname{PMod}\left(S_{0, n}^{1}\right)$ by [34] and [21, Theorem 9.1]. By Hölder's inequality, we may assume $p=1$. The counterpart to Equation (\#) for $p=1$ is given in [7, Equations 8,12-14], and hence Lemma 4.2 again holds verbatim. We remark that, for $p=2$, Equation (\#) is given in [4, Lemma 4]. We will omit the details.

Alternatively, one can combine Theorem 1.1 with the following theorem of Crisp and Wiest to prove Corollary 1.2(2) for $p=2$. A right-angled Artin group is of planar type if the defining graph can be realized as the incidence graph of simple closed curves in the plane [18].

Theorem 4.4 [18, Theorem 12] Every right-angled Artin group of planar type admits a quasi-isometric group embedding into $\left(\operatorname{Symp}\left(D^{2}, \partial D^{2}\right), d_{2}\right)$.

\subsection{Two-generated subgroups of right-angled Artin groups}

Leininger and Margalit showed the following structure theorem for two-generated subgroups of pure braid groups:

Theorem 4.5 [32] Every two-generator subgroup of a pure braid group is either abelian or free. 
In [3], Baudisch established the same fact for two-generated subgroups of right-angled Artin groups. Baudisch's proof uses somewhat involved combinatorial arguments. Combining Leininger and Margalit's result with our main theorem, we obtain a more transparent proof of Baudisch's result:

Proof of Corollary 1.3 Each right-angled Artin group embeds into a pure braid group, in which every two-generated subgroup is either abelian or free.

\subsection{Hyperbolic manifolds}

It is known that every word-hyperbolic Coxeter group is virtually special [23]. Examples of discrete cocompact hyperbolic reflection groups are known to exist up to dimension eight by the work of Bugaenko [10; 11]. By Corollary 1.4, there exist examples of closed hyperbolic $n$-manifolds whose fundamental groups admit quasi-isometric group embeddings into pure braid groups for $n \leq 8$.

More concretely, the commutator group of a right-angled Coxeter group is special [20; 19]. In particular, the commutator subgroup of the reflection group of the allright 120-cell in $\mathbb{H}^{4}$ provides a specific example of a closed hyperbolic 4-manifold group which is special [18]. Theorem 1.1 implies that this 4-manifold group admits a quasi-isometric group embedding into a pure braid group.

For an arbitrary $n$, the existence of closed hyperbolic $n$-manifold groups inside of right-angled Artin groups follows from the more recent work of Bergeron and Wise [6] and Bergeron, Haglund and Wise [5].

\subsection{The isomorphism, conjugacy, and membership problem for finitely presented subgroups of braid groups}

The proofs of Corollaries 1.6 and 1.7 are straightforward from the work of Bridson, after combining it with Theorem 1.1.

Theorem 4.6 [9, Theorems 1.1 and 1.2] There exists a right-angled Artin group $A_{1}$ such that the isomorphism problem for finitely presented subgroups of $A_{1}$ is unsolvable. Furthermore, there exists a right-angled Artin group $A_{2}$ and a finitely presented subgroup $H<A_{2}$ such that the conjugacy and membership problems for $H$ are unsolvable.

Proofs of Corollaries 1.6 and 1.7 Let $A_{1}$ and $A_{2}$ be as in Theorem 4.6. Then Theorem 1.1 implies that $A_{1}$ and $A_{2}$ embed into $B_{n}$ for every sufficiently large $n$. 


\section{References}

[1] I Agol, The virtual Haken conjecture, Doc. Math. 18 (2013) 1045-1087 MR3104553

[2] V I Arnold, B A Khesin, Topological methods in hydrodynamics, Applied Mathematical Sciences 125, Springer, New York (1998) MR1612569

[3] A Baudisch, Subgroups of semifree groups, Acta Math. Acad. Sci. Hungar. 38 (1981) 19-28 MR634562

[4] M Benaim, J-M Gambaudo, Metric properties of the group of area preserving diffeomorphisms, Trans. Amer. Math. Soc. 353 (2001) 4661-4672 MR1851187

[5] N Bergeron, F Haglund, D T Wise, Hyperplane sections in arithmetic hyperbolic manifolds, J. Lond. Math. Soc. 83 (2011) 431-448 MR2776645

[6] N Bergeron, D T Wise, A boundary criterion for cubulation, Amer. J. Math. 134 (2012) 843-859 MR2931226

[7] M Brandenbursky, Quasi-morphisms and $L^{p}$-metrics on groups of volumepreserving diffeomorphisms, J. Topol. Anal. 4 (2012) 255-270 MR2949242

[8] M Brandenbursky, E Shelukhin, On the large-scale geometry of the $L^{p}$-metric on the symplectomorphism group of the two-sphere, preprint (2014) arXiv:1304.7037

[9] M R Bridson, On the subgroups of right-angled Artin groups and mapping class groups, Math. Res. Lett. 20 (2013) 203-212 MR3151642

[10] V O Bugaenko, On reflective unimodular hyperbolic quadratic forms, Selecta Math. Soviet. 9 (1990) 263-271 MR1074386

[11] V O Bugaenko, Arithmetic crystallographic groups generated by reflections, and reflective hyperbolic lattices, from: "Lie groups, their discrete subgroups, and invariant theory", (È B Vinberg, editor), Adv. Soviet Math. 8, Amer. Math. Soc. (1992) 33-55 MR1155663

[12] M Casals-Ruiz, A Duncan, I Kazachkov, Embedddings between partially commutative groups: two counterexamples, J. Algebra 390 (2013) 87-99 MR3072113

[13] R Charney, An introduction to right-angled Artin groups, Geom. Dedicata 125 (2007) 141-158 MR2322545

[14] M T Clay, C J Leininger, J Mangahas, The geometry of right-angled Artin subgroups of mapping class groups, Groups Geom. Dyn. 6 (2012) 249-278 MR2914860

[15] M Clay, C J Leininger, D Margalit, Abstract commensurators of right-angled Artin groups and mapping class groups, Math. Res. Lett. 21 (2014) 461-467 MR3272023

[16] J Crisp, L Paris, The solution to a conjecture of Tits on the subgroup generated by the squares of the generators of an Artin group, Invent. Math. 145 (2001) 19-36 MR1839284

[17] J Crisp, B Wiest, Embeddings of graph braid and surface groups in right-angled Artin groups and braid groups, Algebr. Geom. Topol. 4 (2004) 439-472 MR2077673 
[18] J Crisp, B Wiest, Quasi-isometrically embedded subgroups of braid and diffeomorphism groups, Trans. Amer. Math. Soc. 359 (2007) 5485-5503 MR2327038

[19] M W Davis, The geometry and topology of Coxeter groups, London Mathematical Society Monographs Series 32, Princeton Univ. Press (2008) MR2360474

[20] C Droms, A complex for right-angled Coxeter groups, Proc. Amer. Math. Soc. 131 (2003) 2305-2311 MR1974626

[21] B Farb, D Margalit, A primer on mapping class groups, Princeton Mathematical Series 49, Princeton Univ. Press (2012) MR2850125

[22] F Haglund, D T Wise, Special cube complexes, Geom. Funct. Anal. 17 (2008) 15511620 MR2377497

[23] F Haglund, D T Wise, Coxeter groups are virtually special, Adv. Math. 224 (2010) 1890-1903 MR2646113

[24] M Kapovich, RAAGs in Ham, Geom. Funct. Anal. 22 (2012) 733-755 MR2972607

[25] B Khesin, R Wendt, The geometry of infinite-dimensional groups, Ergeb. Math. Grenzgeb. 51, Springer (2009) MR2456522

[26] S-h Kim, Co-contractions of graphs and right-angled Artin groups, Algebr. Geom. Topol. 8 (2008) 849-868 MR2443098

[27] S-h Kim, T Koberda, Embedability between right-angled Artin groups, Geom. Topol. 17 (2013) 493-530 MR3039768

[28] S-h Kim, T Koberda, The geometry of the curve graph of a right-angled Artin group, Internat. J. Algebra Comput. 24 (2014) 121-169 MR3192368

[29] S-h Kim, T Koberda, An obstruction to embedding right-angled Artin groups in mapping class groups, Int. Math. Res. Not. 2014 (2014) 3912-3918 MR3239092

[30] S-h Kim, T Koberda, Right-angled Artin groups and finite subgraphs of curve graphs, preprint (2014) arXiv:1310.4850 To appear in Osaka J. Math.

[31] T Koberda, Right-angled Artin groups and a generalized isomorphism problem for finitely generated subgroups of mapping class groups, Geom. Funct. Anal. 22 (2012) 1541-1590 MR3000498

[32] C J Leininger, D Margalit, Two-generator subgroups of the pure braid group, Geom. Dedicata 147 (2010) 107-113 MR2660568

[33] L Polterovich, The geometry of the group of symplectic diffeomorphisms, Birkhäuser, Basel (2001) MR1826128

[34] S Smale, Diffeomorphisms of the 2-sphere, Proc. Amer. Math. Soc. 10 (1959) 621-626 MR0112149

[35] D T Wise, The structure of groups with a quasiconvex hierarchy, preprint (2011) Available at http://www.math.mcgill.ca/wise/papers.html 
[36] C Wrathall, The word problem for free partially commutative groups, J. Symbolic Comput. 6 (1988) 99-104 MR961373

Department of Mathematical Sciences, Seoul National University Seoul 151-747, South Korea

Department of Mathematics, University of Virginia Charlottesville, VA 22904-4137, USA

s.kim@snu.ac.kr, thomas.koberda@gmail.com

Proposed: Danny Calegari

Seconded: Leonid Polterovich, Martin R Bridson

Received: 27 May 2014 Revised: 2 February 2015 